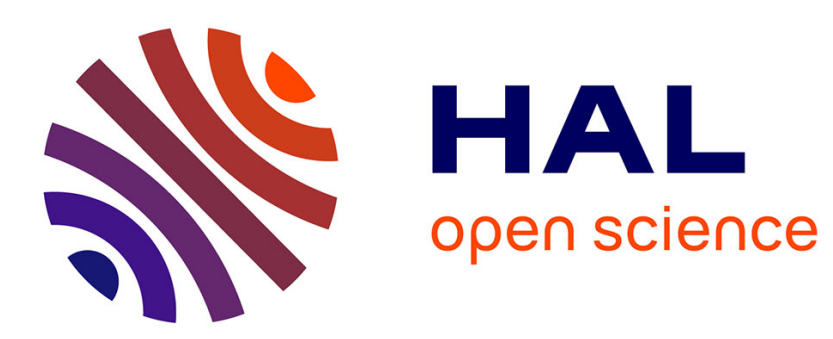

\title{
On the Use of 2D Navigable Maps for Enhancing Ground Vehicle Localization
}

Clément Fouque, Philippe Bonnifait

\section{To cite this version:}

Clément Fouque, Philippe Bonnifait. On the Use of 2D Navigable Maps for Enhancing Ground Vehicle Localization. IROS2009, 2009, United States. pp.887. hal-00445303

\section{HAL Id: hal-00445303 https://hal.science/hal-00445303}

Submitted on 8 Jan 2010

HAL is a multi-disciplinary open access archive for the deposit and dissemination of scientific research documents, whether they are published or not. The documents may come from teaching and research institutions in France or abroad, or from public or private research centers.
L'archive ouverte pluridisciplinaire HAL, est destinée au dépôt et à la diffusion de documents scientifiques de niveau recherche, publiés ou non, émanant des établissements d'enseignement et de recherche français ou étrangers, des laboratoires publics ou privés. 


\title{
On the Use of 2D Navigable Maps for Enhancing Ground Vehicle Localization
}

\author{
Clément Fouque and Philippe Bonnifait \\ Heudiasyc UMR 6599, Université de Technologie de Compiègne, France.
}

\begin{abstract}
For ground vehicle localization, hybrid-GNSS localizers now use commonly dead-reckoning sensors, like odometers or inertial units. They are designed to increase the accuracy, the integrity and the availability of the localization information, particularly in areas where the satellite signals are subject to outages and multipaths. In this paper, a data-fusion method is proposed to take benefits of $2 \mathrm{D}$ navigable road-maps in a tightly-coupled approach. In such a problem, 3D modeling is mandatory to process the pseudo-range information of the satellites. Our proposal is to use a 2D map as a heading measure in a Earth tangential frame. This is called "map-aided odometry". A Kalman filter, gating the normalized innovation signals, is applied to merge the redundant exteroceptive information in a cautious way. Experimental results are reported to quantify the performance gain of the proposed approach relying on the map-aided technique. We show that this fusion scheme increases the accuracy and the availability of the provided localization specially in GNSS-challenging environments.
\end{abstract}

\section{INTRODUCTION}

Nowadays, localization systems in ITS have become familiar with the use of proprioceptive sensors in hybrid Global Navigation Satellite System (GNSS). These sensors, like Wheel Speed Sensors (WSS) or inertial units for instance, can be either loosely-coupled [1] or tightly-coupled [2] with the GNSS measurements. Recently, the use of tightly coupled methods in hybrid GNSS has proved to enhance the performance of the localizer in urban area [2]. Indeed, a tightly-coupled method allows the position computation with less than 4 visible satellites, as the fusion is made at the pseudo-ranges level. Nevertheless, a complete satellite outage remains a challenging situation, as location estimation relies only on proprioceptive measurements.

The use of a navigable road map is a promising technique to tackle this issue. A navigable road-map is a source of a-priory knowledge about the surrounding road network, and the assumption of on-road vehicle is commonly made. Several ways to introduce this knowledge have already been studied. For instance, the road network can constrain the snapshot GNSS computation [3], [4]. Moreover, an accurate $3 \mathrm{D}$ map can also be used to constraint the localization in a dynamic fusion process since it provides altitude information [5], [6] like a Digital Terrain Model [7]. A 2D navigable road map can also be considered as a positioning sensor in complement of a GNSS receiver [1]. Moreover, it can be used as a virtual DGPS base station, providing corrections to the vehicle localizer [8].

Every map-based methods has to handle particular issues when using the map. Firstly, a common assumption is that the vehicle is parallel to the current road segment, and a map-matching step has been done to select the correct road. Numerous map-matching algorithm are available in the literature [9]. In case of mono-hypothesis map-matching, each road junction can be considered as an ambiguous situation. Secondly, these maps are often inaccurate because of a limited spatial-sampling of the road geometry, biases (absolute errors), and absence of altitude information. Thus, using the map for providing a estimated location introduces non-zero mean errors, and computing a map-matched location leads to global estimation errors. Fortunately, map precision is often better than map accuracy. This precision can be exploited for enhancing global localization.

Since 2D navigable road maps are now affordable and already embedded in many navigation systems, we address the problem of taking benefit from this information for enhancing global localization. Therefore, a method to merge a 2D navigable road map with a hybrid GNSS system is presented in this paper. Given the low accuracy of a navigable road map, our proposal uses the road-map as a vector field. Hence, the road-map provides heading information in a Earth-tangential frame, like a compass sensor does. Unfortunately, both the map measurement and the GNSS raw measurements can introduce outliers in the fusion process. According to the mono-hypothesis Kalman filter framework, a cautious strategy, based on gating innovation signals, will be used to reject these outliers. Since error modelling is of particular importance, EGNOS/WAAS corrections are exploited to eliminate as much as possible biases on the pseudo-ranges, and estimate the noise variances.

The paper is organized as follow. First, an hybrid GNSS fusion system is stated. Due to the Kalman filter framework, a state-space description of the system is introduced, including the process model and the observation model of the dead-reckoning measurements and of the GNSS raw measurements. The second section introduces navigable roadmaps and the map observation model will be stated. The next section presents the data-fusion framework. A cautious strategy used to handle GNSS and map outliers is described. Finally, experimental results are given to illustrate the efficiency of the introduced framework, particularly regarding the proposed map-aided odometry.

\section{HYBRIDIZED GNSS SYSTEM}

\section{A. Working frame}

In order to merge GNSS measurements, expressed in a Earth Centered-Earth Fixed (ECEF) frame, and proprioceptive measurements, expressed relatively to the vehicle body 
frame, a local working frame must be defined [10, Chap. 10.4]. For that, a East - North - Up (ENU) navigation frame is used. This frame is tangent to the Earth ellipsoid at a given reference point, close to the working area. This frame is valid only for a limited area and thus, must be updated from time to time. The used reference point is linked to the map. This will be introduced later.

\section{B. Process model}

Let us consider the process model used for the prediction step of a Kalman filter. The process model derives from a vehicle motion model. Hereafter, the vehicle motion is depicted using a differential-drive motion model under the following assumptions:

- Locally planar and locally circular motion at the rearaxle middle point,

- Rigid body and pure rolling.

This motion model is then defined in a local plane. Hence, state components must be added to take into account 3D GNSS measurements. The vehicle altitude must be introduced. Since we considered hereafter navigation areas of little altitude variation, a constant elevation model is used. Moreover, the raw GNSS measurements are biased due to the receiver internal-clock offset. This clock offset must be estimated on line too. To allow this offset to evolves during an outage, the clock drift is also considered. Therefore, the following state vector $X_{k}$ is :

$$
X_{k}=[x, y, z, \psi, v, \omega, d, \dot{d}]
$$

Where $(x, y, \psi)$ describes the 2D vehicle pose, $z$ its altitude, $(v, \omega)$ the linear and angular velocities of the vehicle, and $(d, \dot{d})$ the GNSS receiver clock offset and drift. So, a discrete motion model can be given by, $T_{e}$ being the sampling period:

$$
\left\{\begin{array}{ccc}
x_{k+1} & =x_{k}+T_{e} v_{k} \cos \left(\psi_{k}+\frac{1}{2} T_{e} \omega_{k}\right) \\
y_{k+1} & =y_{k}+T_{e} v_{k} \sin \left(\psi_{k}+\frac{1}{2} T_{e} \omega_{k}\right) \\
z_{k+1}=z_{k} & T_{e} \cdot \omega_{k} \\
\psi_{k+1}=\psi_{k}+ & \\
v_{k+1}=v_{k} & T_{e} \cdot \dot{d}_{k} \\
\omega_{k+1}=\omega_{k} & \\
d_{k+1}=d_{k}+ & \dot{d}_{k}
\end{array}\right.
$$

The vehicle evolution model is then depicted by a set of input-free, non-linear equations, where $\alpha_{k}$ is the process model errors:

$$
X_{k+1}=f\left(X_{k}\right)+\alpha_{k}
$$

\section{Sensors models}

1) Dead-Reckoning: The Dead-Reckonnig (DR) measurements relies on three sensors: a yaw-rate gyro and two rear WSS. They are provided by the ABS system of our experimental vehicle. By neglecting their latency, they provide a direct measurement of the vehicle kinematics. Since DR is crucial in the presented method, a fiber optic gyrometer (KVH400) is used for the yaw-rate sensing. Such a sensor does not require any offset observation process and provides directly the angular velocity of the vehicle body. Its measurement is denoted $\omega_{m}$.

According to assumptions of Section II-B, the rear WSS provide good estimates of the linear speeds of the wheels. With $v_{l}$ (resp. $v_{r}$ ) denoting the left (resp. right) linear wheel speed, the observation model of the DR sensors is given by the following linear equation set:

$$
\left[\begin{array}{c}
v_{l} \\
v_{r} \\
\omega_{m}
\end{array}\right]=\left[\begin{array}{cc}
1 & -\frac{L}{2} \\
1 & \frac{L}{2} \\
0 & 1
\end{array}\right] \cdot\left[\begin{array}{c}
v \\
\omega
\end{array}\right]
$$

Where $L$ is the rear axle length.

This formalization allows isolating the sensor measurements which can be useful for a fault detection and isolation strategy.

2) GNSS measurements: The GNSS raw measurements are provided by a Septentrio PolarX2e GPS receiver, working at $10 \mathrm{~Hz}$. We have chosen to use only the L1 pseudo-ranges (PR). The measured PR are estimated by the receiver regarding the time of flight of the signal through the atmosphere [10]. So, the PR observation model for a given satellite (denoted $s$ ) is given by:

$$
\rho_{m}=R+c\left(d t-d t_{s}\right)-\delta_{\mathrm{atm}}-M
$$

With:

- $\rho_{m}$ : measured PR

- $R$ : true geometrical distance between the observed SV and the receiver,

- $d t_{s}$ : Space Vehicle (SV) clock offset,

- $\delta_{\text {atm }}$ : elongation due to atmospheric effects,

- $M$ : multipath error.

Fortunately, some assumptions are often made to simplify this general model. First, let us assume that the signal is not affected by multipath (it is the case in the reported experiments). Second, the SV clock offset is estimated using the broad-casted ephemeris. Third, Satellite Based Augmentation Systems (SBAS) like WAAS/EGNOS broad-casted information is available. With these corrections (fast and longterm, SV clock bias, ionosphere and troposphere), corrected pseudo-range errors are assumed to be zero-mean, Gaussian and uncorrelated [11]. The PR error variance (denoted $\sigma_{\rho}$ ) is also provided by these corrections. Therefore, the PR observation model is reduced to:

$$
\rho=R+d+\beta_{\rho}
$$

Where $\beta_{\rho}$ represents the resulting noise with a covariance matrix $Q_{\rho}$. Assuming $n$ visible $\mathrm{SV}$, the observation model is non-linear and non-stationary:

$$
\left\{\begin{array}{l}
\rho^{i}=h^{i}\left(X_{k}\right)+\beta_{k}^{i} \\
Q_{\rho}^{i, i}=\sigma_{\rho}^{i}
\end{array} \quad \forall i=1, \ldots, n\right.
$$


$Q_{\rho}^{i, i}$ denotes the $i^{t h}$ diagonal term of the noise covariance matrix $Q_{\rho}$.

\section{NAVIGABLE ROAD MAPS}

\section{A. Description}

Commonly, a road database contains a vectorial description of the road network where each road is defined as an object. A road object in the database is described by both geometrical and topological information and is identified by a single identification (Id).

Each road is spatially sampled and described by a set of shape points and two nodes (origin and end). These points form a polygonal curve describing the shape of the road center-line. The number of carriageways and lines width are also often known. In addition, a connectivity table is given, allowing the user to known which roads are connected with a given Id. This connectivity table is usually used for path planning. It can also improve the tracking of the roads [12].

Unfortunately, a digital road map is subject to bad precision (ie. low spatial sampling) and bad accuracy (ie. absolute offsets). Therefore, using the road shape for constraining the position estimation induces non zero-mean errors which can affect badly any Bayesian estimation process. Since the precision of the map is often better than its accuracy, we suggest to use the heading information provided by the polygonal curve describing the road, under the hypothesis that the car heading is parallel to the road. The DR process can take advantage of this information, as its drift is very dependent of the quality of the heading estimate [13].

\section{B. Road Cache}

A complete navigable road map represents a huge amount of data that requires high computation capabilities, even for offline computation. According to a real-time context, a reduced road cache is extracted around a given point from the database [12]. This given point is chosen as the origin of the local tangent frame (see Section II-A). Thus, the working frame must be updated when a new road cache is requested. The coordinates of the nodes and shape points are then converted into the working frame. Finally, during the extraction, several road features, such as road segment heading or length, are computed in order to accelerate the map-matching computation. This is a second advantage of using a road cache.

\section{Map observation}

In order to handle unavoidable position offsets, the map observation itself is quite simple since it is only a heading information, according to the driving direction. Let suppose for simplification that the right segment has been map-matched. By denoting $\psi_{r}$ this segment heading, the observation model used for map update is:

$$
\psi_{r}=\psi_{k}+\beta_{\psi}
$$

Where $\beta_{\psi}$ is the heading error.

As a Kalman Filter is used, the measurement variance must be known. Considering that the vehicule can maneuver at low speed, this variance is computed according to vehicule velocity $v$ and the road width $W$. By modeling the vehicle trajectory by a triangular wave on the road, we can compute the maximum deviation angle $\xi$ between the vehicle heading and the segment heading. $\xi$ is given by:

$$
\begin{cases}\sin \xi=\frac{W}{v} & \text { if } v \geq W \\ \sin \xi=1 & \text { else }\end{cases}
$$

Taking $\xi$ as the $3 \sigma$ confidence zone for the map observation $\psi_{r}$, we are now able to quantify the variance (denoted $\sigma_{r}$ ) for the map observation:

$$
\sigma_{r}=\frac{\xi}{3}
$$

Equation (10) relaxes the hypothesis of having a heading strictely parrallel to the road at low speeds.

\section{GLOBAL FUSION ALGORITHM}

We present in this section a cautious strategy for tightlycoupling raw GNSS measurements with 2D geographic information and DR measurements. We suppose here that the DR measurements are trustworthy. This an important parameter since the DR estimate has to be reliable in our data fusion process. So, the fusion of the DR sensors with the predicted state is always carried out (see Fig.1). On the opposite, we consider that both the GNSS measurements and the map heading measure can be defective. Indeed, GNSS signals can suffer from multipath or other propagation troubles, and the map-matching stage can provide a wrong road, leading to a faulty map measurements for example. Before doing the data fusion of the prediction with the exteroceptive information, validations of the map heading and of the GNSS PR are done by checking the consistency of the innovations of the Kalman filter.

\section{A. GNSS validation process}

A validation stage is made on the GNSS PRs to avoid the fusion of biased measurements. This validation is made before the second state update (Fig.1). The gating of the measurements is done using the Normalized Innovation Squared (NIS) test that uses the estimated state $<$ $X_{k \mid k-1, D R}, P_{k \mid k-1, D R}>$ coming from the odometric update. The NIS is given by:

$$
\eta_{k}=\frac{1}{2} \nu_{k} \cdot Q_{\nu}^{-1} \cdot \nu_{k}
$$

Where $\nu$ is the innovation and $Q_{\nu}$ its covariance matrix. The innovation vector is the difference between the current PRs vector $\rho_{k}$ and the predicted measurement corresponding to the predicted state $X_{k \mid k-1, D R}$ :

$$
\nu_{k}=\rho_{k}-h\left(X_{k \mid k-1, D R}\right)
$$

Where $h\left(X_{k \mid k-1, D R}\right)$ is the non-linear observation model derived from the GNSS observation model (6).

In case of an Extended Kalman Filter, the innovation covariance matrix is given by: 


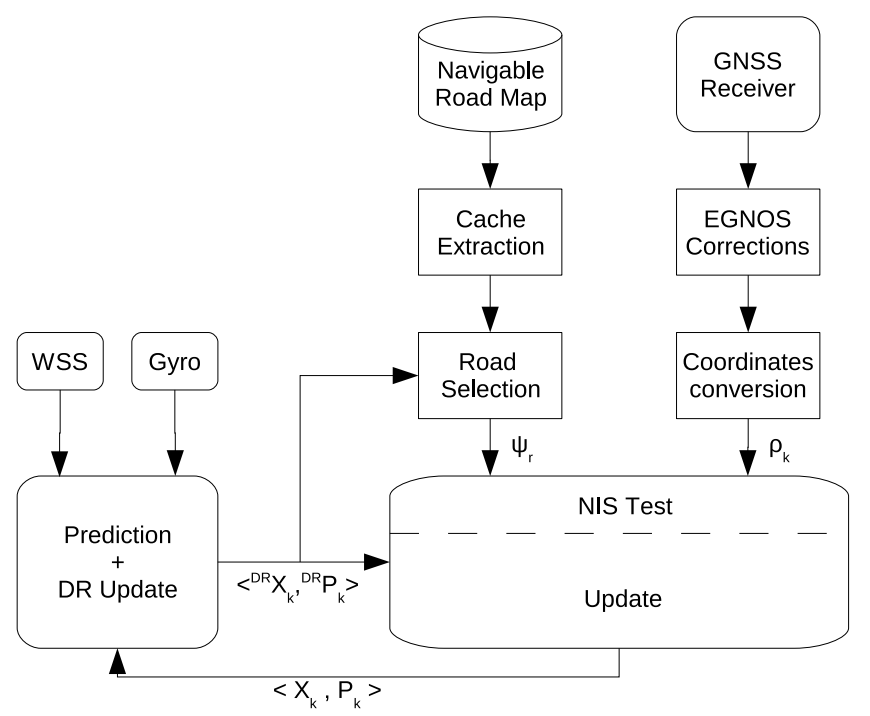

Fig. 1. Algorithm flowchart.

$$
Q_{\nu}=H_{k} \cdot P_{k \mid k-1, D R} \cdot H_{k}^{T}+Q_{\rho}
$$

Where $H_{k}$ is the Jacobian matrix of the PR observation model.

Based on the Gaussian assumption underlying the Kalman filter theory, normally-distributed errors, with zero-mean, are assumed. Therefore, the NIS has to follow a $\chi^{2}$ distribution under normal operation. A threshold value is computed using the $\chi^{2}$ distribution given a False Alarm probability. If the normalized innovation $\eta_{k}$ is greater than this threshold, the measurement is not consistent with the noise model with a high probability [14]. In this case, the PR is rejected.

\section{B. Cautious Map-matching strategy}

In opposition to GNSS that provides distinguished measurements (ie each measurement is associated with a satellite), the use of a navigable map involves a data association step called map-matching. Many map-matching methods have been studied in loosely coupled schemes [9]. It is well known that every method can lead to miss-matching, particularly if a mono-hypothesis strategy is used. As we do mono-hypothesis selection here, the consistency of every selected road segment has to be assessed relatively to the DR state. The most-likely segment is selected as follow. For every segment in the road cache, a selection criterion is computed according to an estimation of the vehicle 2D pose after the DR update. In this algorithm, the mapmatching is state-based, contrary to the measurement-based method presented in [3]. It allows using the map even when the GNSS satellites are totally blocked. The selection is done using a normalized Mahalanobis distance based on the vehicle-to-segment distance $d$ and the angular distance $\Delta \psi$ between the road segment and the vehicle current pose, in a similar way than in [1]:

$$
\Delta_{r}=\frac{d^{2}}{\sigma_{d}^{2}+\lambda_{\max }^{2}}+\frac{\Delta \psi^{2}}{\sigma_{r}^{2}+\sigma_{\psi}^{2}}
$$

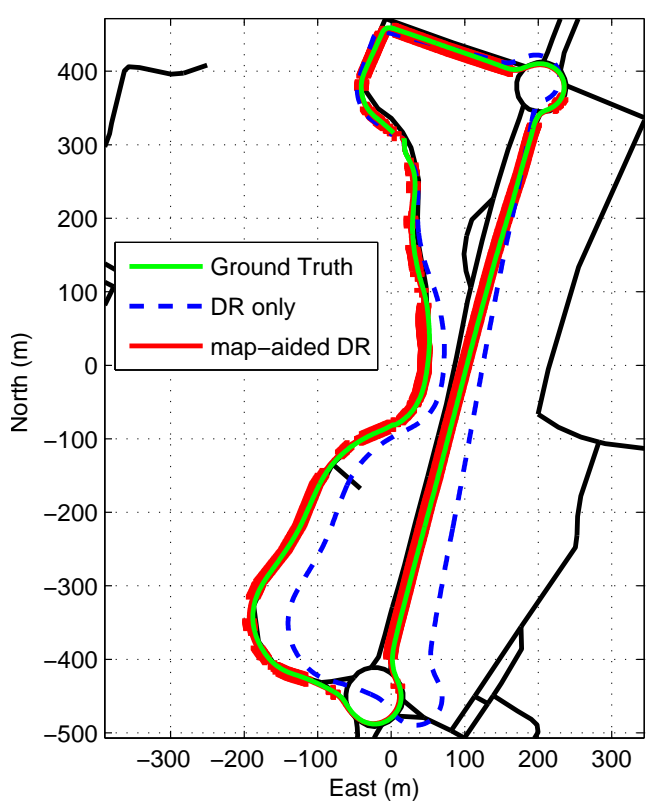

Fig. 2. ENU estimated trajectory using map-aided DR and pure DR.

Where $\sigma_{d}^{2}$ and $\sigma_{r}^{2}$ are the lateral variance and heading variance associated with the road segment. $\sigma_{\psi}$ is the estimated variance of vehicle heading and $\lambda_{\max }$ is the maximum eigen value of the $2 \mathrm{D}$ position covariance. Next, the most likely segment is chosen as the one with the lowest $\Delta_{r}$. Similarly to the GNSS validation stage, the normalized innovation of the most-likely segment is compared to a threshold, computed again using a $\chi^{2}$ distribution. If $\Delta_{r}$ is greater than this threshold, the selected road-segment is considered as inconsistent and the estimated state is not map-updated. This improves the internal integrity of the method [15]. For instance, if a road cache extraction error occurs, the map will be always rejected.

The estimated variance of the position influences also the map usage. If the position variance is small, the algorithm tends to reject any incoherent map information, whereas the map is used more often if the position variance increases. This remark illustrates an additional risk due to the monohypothesis approach: road junctions create ambiguous areas where the risk of miss-matching drastically increases. To prevent from this, ambiguity zones are defined around every road junction in the road cache. The size of these zones depends on the map quality. In practice, when the vehicle is located within an ambiguity area, the map is considered as unavailable.

\section{Kalman-based algorithm}

The global fusion process relies on a mono-hypothesis Extended Kalman Filter (EKF) using serialized state estimation stages. At a first step, a predicted state $X_{k \mid k-1}$ is computed using (3). Then, a first state estimate $X_{k \mid k, D R}$ is computed using DR measurements (4). After this stage, map data and GNSS measurements are treated simultaneously. EGNOS corrections are applied to the PRs, and finally the 

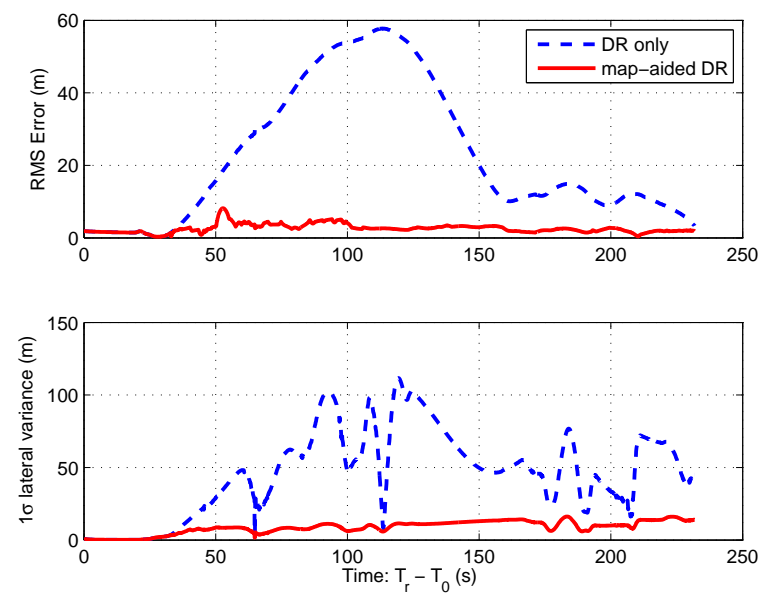

Fig. 3. RMS error and $1 \sigma$ lateral uncertainty for map-aided DR and pure DR.

consistency of the PR vector is tested according to (11). For the map measurement, we first check if the vehicle is located within an ambiguous area. If the map is available, then the road selection is made according to (14). After that, the DRestimated state is updated with the map heading and/or with the PRs, or not updated at all if both are rejected.

This algorithm is well adapted to asynchronous sensor fusion, as each update stage is conducted separately. With an efficient implementation of the map-matching method, the map update can be realized at the DR sensor frequency, where as GNSS correction can be done at a lower frequency.

\section{EXPERIMENTAL RESULTS}

Experiments have been carried out in May 2007 using an experimental vehicle of the lab Heudiasyc. The reference path of the vehicle has been recorded using a Trimble 5700 DGPS PPK receiver and 4 base stations from the French Orpheon network. The car was driven in Compiegne suburbs, which constitute an open area with occasional tree foliage leading to GPS errors and bad geometrical configuration of the SV constellation. For all the following experiments, a unique road cache was used with the reference point located at N $49^{\circ} 23^{\prime} 6.36 ", \mathrm{E} 2^{\circ} 47^{\prime} 2.04 "$. The GPS raw data were recorded using a Septentrio PolarX2e at $10 H z$.

\section{A. Map-aided DR}

In this first experiment, the benefit of the map heading information on the DR estimates is shown. GPS is used up to the first vehicle displacement for filter initialization purposes. The initialization stops after $45 s$. Fig. 2 depicts the vehicle trajectory in the working frame. It also shows the estimated trajectories using only the DR measurements (dashed-blue), the map-aided DR estimates (red-solid) with an emphasis on the locations where the map information was used in the position computation (red-stars). Fig.3 points out the RMS error for the DR-only and map-aided DR estimates, and the $1 \sigma$ lateral uncertainty of vehicle estimated location.
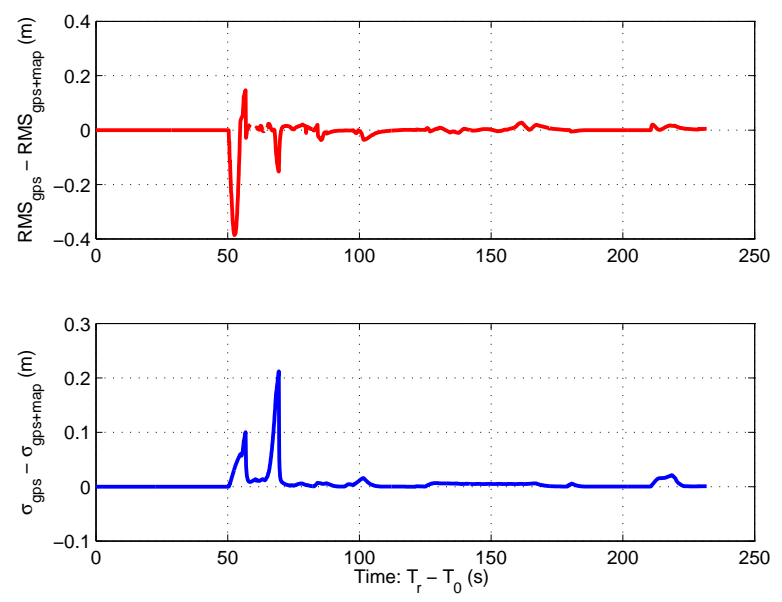

Fig. 4. RMS errors of hybridized GPS and Map-aided hybridized GPS. The difference on the estimated standard deviation is also reported.

According to Fig.2, it can be clearly seen that the estimated heading after initialization is not precisely estimated. This poor initialization introduces a small initial heading error into the DR-only process. When using the map to correct DR estimates, this offset is reduced allowing a much better estimation of the vehicle localization. In this test, the map was used about $49 \%$ of the time, including the initialization stage. The gain on the estimation process is clearly visible on Fig.3, as the RMS error stays under $6 \mathrm{~m}$. The estimated lateral variance for the map-aided also shows significant improvement as it stands under $25 \mathrm{~m}$. The fluctuation of the lateral accuracy are due to the heading angle variations which modifies the steady state behaviour of the Kalman Filter [16].

According to these results, we can conclude that mapaided DR is useful to recover from initialization errors, which frequently occurs at the beginning of an outage. Moreover, when using low-cost DR sensors, map-aiding odometry is useful to handle longer outages with less drift. In that case, the accuracy of the vehicle estimates degrades slower than with DR only systems.

\section{B. Map-aided hybridized GPS}

In this second experiment, the emphasis is made on the neutrality of map-aiding under good GNSS signal conditions. Indeed, a well-tuned multisensor fusion technique has to merge all the available information. When GNSS is running with a good satellite configuration, the use of the map must not degrade the performance. Often, because of the difficulty to merge low-quality data with GNSS, aiding-systems are mainly used as backup system into [1]. We have noticed that our data fusion approach gives the same results when GPS is available with or without the map. To evalute this, let us consider the difference between RMS error for hybridized GPS and for map-aided GPS under identical GPS signal conditions. This is shown in Fig.4. A positive value means that the RMS error for hybridized GPS without map-aiding 

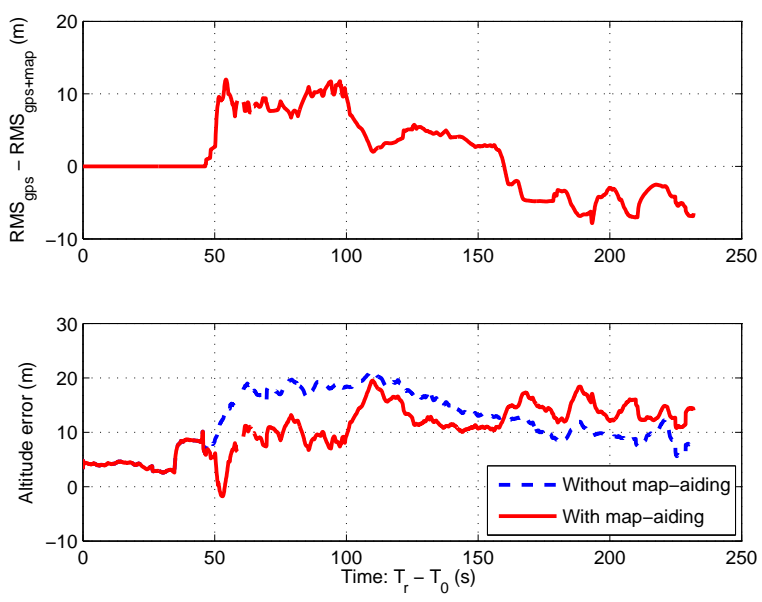

Fig. 5. Quality of the estimation using only two satellites

is greater than the RMS error of map-aided hybridized GPS estimates. The $1 \sigma$ variance difference is also given in Fig.4. Peaks are due to GPS errors.

According to the upper curve, the difference mean-value is $3 \mathrm{~mm}$ for the RMS error between hybrid GNSS and mapaided hybrid GNSS. Likewise, the difference mean-value is $6 \mathrm{~mm}$ for the estimated variance. According to these two results, neither improvement nor degradation can be seen when the GNSS receiver is under good signal conditions. It should be noticed that the map is used $33 \%$ of time in this test. This is an interesting feature that proves the efficiency of the proposed fusion method. So, the map-aiding can be used continuously since it gives similar results under good signal conditions.

\section{Degraded GPS signal conditions}

For this final experiment, the GPS availability is intentionally degraded: Only the two highest SV were considered as visible (PRN 1 and PRN 11). In such a situation similar to an urban canyon, a standalone GPS receiver is not able to provide a positioning solution. We compare the performance of the hybridized GPS system with and without the map. Fig. 5 shows results of this experiment. The upper chart shows the difference between the RMS error of the hybridized GPS and the one of the map-aided hybridized GPS. The lower chart depicts the estimated elevation error for both systems.

After the initialization stage, one can notice that the hybridized GPS without map-aiding suffers from a bigger error. The mean-value of this difference is around $1.5 \mathrm{~m}$. The accuracy of the estimated state is so improved. Considering the elevation deviation of both systems, we can notice that the map-aided hybridized GPS suffers from a lower altitude deviation. The mean value for the map-aided hybridized GPS is $10.65 m$ whereas the mean-value for the hybridized GPS is only $12.02 \mathrm{~m}$. We can also establish a relationship between the elevation difference $(1.36 \mathrm{~m})$ and the RMS error difference $(1.5 \mathrm{~m})$. Under harsh reception conditions, the estimation of the elevation is logically degraded.

\section{CONCLUSION}

In this paper, a map-aiding technique for hybrid GNSS systems has been presented. The proposed approach uses only $2 \mathrm{D}$ maps in a $3 \mathrm{D}$ localization problem, thanks to the use of an adequately chosen local frame and a road cache data set. In this frame, the data fusion is easy to perform. After the updating with the DR measurements, the map heading is obtained after a road selection. Afterward, the estimated state is corrected by the map heading. When the system suffers from GPS outages, the map-aiding odometry allows compensating vehicle heading errors.

The proposed data fusion scheme based on the detection and the rejection of doubtful exteroceptive information compared to the estimated movement of the vehicle has proved good qualities. The map can be always fused, DR drift is compensated and the GNSS measurements can be used even when less than 4 satellites are visible. The experimental results have shown that the use of a $2 \mathrm{D}$ navigable map in an hybridized GPS system improves the 2D estimates. Unfortunately, under challenging conditions, the altitude is poorly estimated. Therefore, the use of future 3D navigable maps constitutes the main perspective of this research.

\section{REFERENCES}

[1] A. Lahrech, C. Boucher, and J.-C. Noyer, "Accurate vehicle positioning in urban areas," in IECON 2005, 2005.

[2] U. Bhatti, W. Ochieng, and S. Feng, "Integrity of an integrated GPS/INS system in the presence of slowly growing errors. part i: A critical review," GPS Solutions, vol. 11, no. 3, pp. 173-181, July 2007.

[3] C. Fouque and P. Bonnifait, "Tightly coupled GIS data in GNSS fix computation with integrity test," IJIIDS, vol. 2, no. 2, pp. 167-186, 2008.

[4] Y. Cui and S. Ge, "Autonomous vehicle positioning with GPS in urban canyon environments," IEEE Trans. on Rob. and Aut., vol. 19, pp. 1525, 2003.

[5] D. Betaille, R. Toledo-Moreo, and J. Laneurit, "Making an enhanced map for lane location based services," in ITSC 2008, 2008, pp. 711716.

[6] L. Kang, T. Han-Sue, and J. Hedrick, "An enhanced gps-based vehicle positioning system through sensor fusion of digital map data," in AVEC'08, 2008.

[7] J. Li, G. Taylor, and D. B. Kidner, "Accuracy and reliability of mapmatched GPS coordinates: the dependence on terrain model resolution and interpolation algorithm," Computers \& Geosciences, vol. 31, no. 2, pp. $241-251,2005$.

[8] G. Taylor and G. Blewitt, "Virtual differential GPS and road reduction filtering by map matching," ION'99, pp. 1675-1684, 1999.

[9] M. A. Quddus, W. Y. Ochieng, and R. B. Noland, "Current mapmatching algorithms for transport applications: State-of-the art and future research directions," Transportation Research Part C: Emerging Technologies, vol. 15, no. 5, pp. 312 - 328, 2007.

[10] B. Hofmann-Wellenhof, H. Lichtenegger, and J. Collins, GPS Theory and Practice, 1994.

[11] RTCA/DO-229C, "Minimum operational performance standards for GPS/WAAS airborne equipment," Tech. Rep., 2001.

[12] P. Bonnifait, M. Jabbour, and G. Dherbomez, "Real-time implementation of a GIS-based localization system for intelligent vehicles," EURASIP Journal on Embedded Systems, pp. 12-24, June 2007.

[13] A. Kelly, "Some useful results for closed-form propagation of error in vehicle odometry," CMU-RI-TR-00-20, Tech. Rep., 2000.

[14] M. A. Sturza and A. K. Brown, "Comparison of fixed and variable threshold raim algorithms," ION'90, pp. 437-442, 1990.

[15] H. Sun and M. E. Cannon, "Reliability of an ITS navigation system," ITSC'97, pp. pp. 1040-1046, November 9-11 1997.

[16] B. Mourllion, D. Gruyer, and A. Lambert, "Variance behavior and signification in probabilistic framework applied to vehicle localization," in IEEE/ITSS IV2006, June 2006, pp. 294-299. 